\title{
Nonspecific Interactions Between Proteins and Charged Biomolecules in Electrospray Ionization Mass Spectrometry
}

\author{
Nian Sun, Naoto Soya, Elena N. Kitova, and John S. Klassen \\ Alberta Ingenuity Centre for Carbohydrate Science and Department of Chemistry, University of Alberta, \\ Edmonton, Alberta, Canada
}

An investigation of the nonspecific association of small charged biomolecules and proteins in electrospray ionization mass spectrometry (ES-MS) is described. Aqueous solutions containing pairs of proteins and a small acidic or basic biomolecule that does not interact specifically with either of the proteins were analyzed by ES-MS and the distributions of the biomolecules bound nonspecifically to each pair of proteins compared. For the basic amino acid arginine and the peptide RGVFRR, nonequivalent distributions were measured in positive ion mode, but equivalent distributions were measured in negative ion mode. In the case of uridine $5^{\prime}$-diphosphate, nonequivalent distributions were measured in negative ion mode, but equivalent distributions observed in positive ion mode. The results of dissociation experiments performed on the gaseous ions of the nonspecific complexes suggest that the nonequivalent distributions result from differences in the extent to which the nonspecific complexes undergo in-source dissociation. To test this hypothesis, the distributions of nonspecifically bound basic molecules measured in the presence of imidazole, which protects complexes from in-source dissociation, were compared. In all cases, equivalent distributions were obtained. The results indicate that nonspecific binding of charged molecules to proteins during ES is a statistical process, independent of protein structure and size. However, the kinetic stabilities of the nonspecific interactions are sensitive to the nature of the protein ions. It is concluded that the reference protein method for correcting ES mass spectra for nonspecific ligand-protein binding can be applied to the analysis of ionic ligands, provided that in-source dissociation of the nonspecific interactions is minimized. (J Am Soc Mass Spectrom 2010, 21, 472-481) (C) 2010 American Society for Mass Spectrometry

$\mathrm{E}$ lectrospray ionization mass spectrometry (ESMS) has emerged as a valuable tool for characterizing noncovalent protein-ligand complexes, including antibody-antigen, enzyme-substrate, and lectin-carbohydrate complexes, in vitro [1-3]. In addition to providing a direct and general method for detecting specific protein-ligand complexes in solution, ES-MS can provide quantitative insights into the thermodynamic parameters for protein-ligand binding [4-6]. The ES-MS assay is based on the direct detection and quantification of free and ligand-bound protein ions. For example, the binding constant $\left(\mathrm{K}_{a}\right)$ for a 1:1 protein-ligand complex (PL) is determined from the ratio $(R)$ of the total ion abundance $(A b)$ of bound and unbound protein ions (eq 1) measured by ES-MS for solutions of known initial concentrations of protein $\left([\mathrm{P}]_{\mathrm{o}}\right)$ and ligand $\left([\mathrm{L}]_{\mathrm{o}}\right)$, eq 2:

Address reprint requests to Dr. J. S. Klassen, Department of Chemistry, University of Alberta, Edmonton, Alberta T6G 2G2, Canada. E-mail: john.klassen@ualberta.ca.

$$
\begin{gathered}
\frac{[P L]_{e q}}{[P]_{e q}}=\frac{\sum_{n} A b\left(P L^{n+/-}\right)}{\sum_{n} A b\left(P^{n+/-}\right)}=R \\
K_{a}=\frac{R}{[L]_{o}-\frac{R}{1+R}[P]_{o}}
\end{gathered}
$$

An underlying assumption in the ES-MS assay is that the ion abundance ratio determined in the gas phase is equivalent to the equilibrium concentration ratio in solution, (eq 1). In practice, however, deviations may occur due to non-uniform ionization and detection efficiencies (i.e., response factors) [7], nonspecific proteinligand association during the ES process (i.e., nonspecific binding) $[6,8]$ and gas-phase dissociation (i.e., in-source dissociation) [6,9-11]. Generally, when the ligand is small compared with the protein, such that the size and surface properties of the free and ligand-bound protein are similar, the ion abundance ratio determined in the gas phase is representative of the equilibrium concentration ratio [4-6]. Collision-induced dissocia- 
tion of the gaseous protein-ligand complexes in the ion source is undesirable since it will alter the relative abundance of bound and unbound protein ions. The influence of in-source dissociation on the measured ratio of bound-to-unbound protein depends on the configuration of the ion source, the instrumental conditions used, and the gas-phase stability of the complexes being investigated. While there are few quantitative data reported for the gas-phase stability of proteinligand complexes, available data suggest that complexes that are stabilized predominantly by nonpolar interactions in solution exhibit low gas-phase stabilities and are prone to in-source dissociation, even under very gentle sampling conditions [9, 12]. Protein complexes stabilized through ionic interactions (i.e., saltbridges) also tend to be susceptible to gas-phase dissociation [11], although examples to the contrary are known [13]. In contrast, complexes stabilized by multiple hydrogen bonds in solution tend to resist in-source dissociation [14]. In cases where the gaseous complexes are susceptible to in-source dissociation, the extent of dissociation may be reduced through the addition of a stabilizing additive to the ES solution or to the gasphase $[11,15]$. In contrast, free ligand molecules present in solution may bind nonspecifically to proteins and protein complexes during the ES process. The occurrence of this so-called "nonspecific" ligand binding obscures the true binding stoichiometry in solution and introduces errors in the $\mathrm{K}_{\mathrm{a}}$ values derived from ES-MS measurements [6]. Generally, the formation of nonspecific protein-ligand complexes can be minimized by limiting the initial concentration of the ligand. However, for very weak protein-ligand interactions $\left(\mathrm{K}_{\mathrm{a}}<\right.$ $10^{4} \mathrm{M}^{-1}$ ), high ligand concentrations are required to produce detectable levels of complex. In such cases, nonspecific ligand binding is often unavoidable in ES-MS analysis.

Recently, our laboratory developed two different experimental strategies for identifying the formation of nonspecific protein-ligand complexes during ES-MS analysis. The reporter molecule method $\left(\mathrm{M}_{\text {rep }}\right)$ is a qualitative method that can be used to identify differences in ES droplet histories for proteins and their noncovalent complexes (e.g., protein-ligand and multisubunit protein complexes) [16, 17]. The method involves the addition of a reporter molecule $\left(\mathrm{M}_{\text {rep }}\right)$, which does not bind specifically to the protein or proteinligand complexes of interest, to the ES solution at relatively high concentration (typically $>100 \mu \mathrm{M}$ ). The high concentration promotes the formation of nonspecific interactions between $\mathrm{M}_{\text {rep }}$ and any protein or protein complex present in the ES droplets. From the measured distributions of nonspecifically bound $\mathrm{M}_{\text {rep' }}$ it is possible to establish whether a given protein complex originates in solution or whether it forms, at least in part, from nonspecific binding during the ES process. Complexes originating from nonspecific interactions will necessarily have droplet histories different from those of the unbound protein and specific protein complexes; the nonspecific protein complexes are formed later in the ES process, from older and more concentrated ES droplets. These older droplets will be more concentrated in protein, as well as $\mathrm{M}_{\text {rep }}$. As a result, the distributions of nonspecifically bound $M_{\text {rep }}$ molecules observed for the unbound protein (if present) and specific protein complex(es) will differ from those observed for the nonspecific complexes-the nonspecific complexes will experience more extensive nonspecific binding to $\mathrm{M}_{\text {rep }}$.

A second approach, the reference protein method, involves the addition of a reference protein $\left(\mathrm{P}_{\text {ref }}\right)$, which does not bind specifically to the protein or ligand of interest, in the ES solution [18]. The occurrence of nonspecific protein-ligand binding is identified from the appearance of ions corresponding to nonspecific complexes of $\mathrm{P}_{\text {ref }}$ with one or more ligand molecules. Additionally, the fraction of $\mathrm{P}_{\text {ref }}$ undergoing nonspecific ligand binding can provide a quantitative measure of the contribution of nonspecific ligand binding to the measured abundance of protein and specific proteinligand complex. As a result, errors in binding stoichiometry and $\mathrm{K}_{a}$ introduced by nonspecific ligand binding can be corrected. To date, the $\mathrm{P}_{\text {ref }}$ method has been used primarily for the quantification of proteincarbohydrate interactions by ES-MS [19, 20].

An underlying assumption of the $P_{\text {ref }}$ method is that nonspecific ligand binding during the ES process is independent of the size and structure of the protein species present in the ES droplets. This assumption has been rigorously validated in the case of neutral carbohydrates [18] but not in the case of acidic or basic carbohydrates or other ionic ligands. As a result, the suitability of the $\mathrm{P}_{\text {ref }}$ method for quantifying nonspecific interactions between proteins and charged ligands is unclear. In fact, it was recently reported by Zenobi and coworkers that the $\mathrm{P}_{\text {ref }}$ method failed to properly account for the nonspecific binding of basic peptide ligands to proteins in ES-MS [21].

Here, we describe the first comprehensive study of the nonspecific association of small acidic and basic biomolecules to proteins during ES-MS analysis. Importantly, it is shown that in a given ES-MS experiment, nonspecific binding of biomolecules to proteins during ES is a statistical process, independent of protein structure and size. These findings confirm that the $P_{\text {ref }}$ method can be used to quantify the nonspecific association of ionic ligands to proteins in ES-MS. However, time-resolved gas-phase dissociation experiments revealed that the kinetic stabilities of the nonspecific complexes are sensitive to the nature of the protein and the original distributions of nonspecifically bound charged molecules produced during the ES process may be altered by in-source dissociation. As a result, the successful application of the $P_{\text {ref }}$ method requires that in-source dissociation of the nonspecific interactions be avoided. 


\section{Materials and Methods}

\section{Proteins and Ligands}

The carbohydrate-binding antibody single chain fragment, scFv (26 $539 \mathrm{Da})$, was produced using recombinant technology [22]. The scFv was concentrated and dialyzed against deionized water using microconcentrators (Millipore Corp., Bedford, MA, USA) with a molecular weight cut-off of $10 \mathrm{kDa}$, and lyophilized. The $\mathrm{scFv}$ was weighed immediately after removing it from the lyophilizer, dissolved in a known volume of aqueous $50 \mathrm{mM}$ ammonium acetate, and stored at $-20^{\circ} \mathrm{C}$ until used. Bovine ubiquitin, Ubq (8 $\left.565 \mathrm{Da}\right)$, chicken egg white lysozyme, Lyz (14 $315 \mathrm{Da})$, uridine 5 '-diphosphate (UDP) (2), and arginine (3), were purchased from Sigma-Aldrich Canada (Oakville, ON, Canada) and were used without further purification. The trisaccharide 6'-sialyllactose (1) was purchased from IsoSep $A B$ (Uppsala, Sweden) and the peptide RGVFRR (4) was purchased from BACHEM Bioscience Inc. (King of Prussia, PA, USA); both were used without further purification. The ES solutions were prepared by mixing known amounts of the protein and ligand stock solutions. A $50 \mathrm{mM}$ aqueous solution of ammonium acetate was added to yield a final concentration of 3-7 $\mathrm{mM}$ and $\mathrm{a} \mathrm{pH}$ of $6.5-7.5$.

\section{Mass Spectrometry}

All experiments were performed on a 9.4 tesla Apex II Fourier transform ion cyclotron resonance (FT-ICR) mass spectrometer (Bruker, Billerica, MA, USA) equipped with a nanoflow ES ion source. Descriptions of the instrument and the experimental and instrumental parameters used in the ES-MS measurements, as well as the blackbody infrared radiative dissociation (BIRD) experiments, are given elsewhere $[6,8]$.

\section{Results and Discussion}

To test the suitability of the $P_{\text {ref }}$ method for identifying and quantifying nonspecific protein-ligand interactions involving charged ligands, a series of control experiments were performed on solutions containing pairs of proteins and a small acidic or basic biomolecule, which served as a "non-interacting" charged ligand. The three proteins, $\mathrm{Ubq}$, Lyz, and $\mathrm{scFv}$, served as the model proteins for this study. The basic amino acid arginine (3) and the basic peptide RGVFRR (4) served as non-interacting basic ligands and the weak acids $6^{\prime}$-sialyllactose (1) and UDP (2) served as the non-interacting acidic ligands. ES-MS measurements were performed in both positive and negative mode and the distributions of acidic or basic molecules bound nonspecifically to each protein within a pair were compared.

\section{Acidic Molecules}

The $\mathrm{P}_{\text {ref }}$ method was initially developed to quantify the occurrence of nonspecific binding of neutral carbohydrates to proteins during ES-MS analysis. The method has been extensively tested in positive ion mode and the distributions of nonspecifically bound carbohydrates in a given experiment shown to be independent of the structure and size of the protein [18]. To test whether acidic carbohydrates exhibit behavior similar to that of neutral carbohydrates, ES-MS measurements were performed on solutions containing 1, at elevated concentration, and pairs of the model proteins. The $\mathrm{pK}_{\mathrm{a}}$ of the carboxylic moiety of $\mathbf{1}$ is $\sim 4$ [23] and, at neutral $\mathrm{pH}$, essentially all of $\mathbf{1}$ is deprotonated. Shown in Figure 1 are illustrative ES mass spectra acquired in positive and negative ion modes for solutions of $\mathbf{1}(>100 \mu \mathrm{M})$ with $\mathrm{Ubq}$ and $\mathrm{scFv}$ or Lyz and $\mathrm{scFv}$. In all cases, ions corresponding to free protein and protein bound to one or two molecules of $\mathbf{1}$ were detected. In positive ion mode, the protein and the nonspecific complexes were detected predominantly as their protonated ions, i.e., $(\mathrm{P}+\mathrm{nH})^{n+} \equiv \mathrm{P}^{n+}$ and $(\mathrm{P}+\mathbf{1}+\mathrm{nH})^{n+} \equiv(\mathrm{P}+\mathbf{1})^{n+}$, at charge states $n=5$ and $6(\mathrm{Ubq}), 7-9$ (Lyz) or 8-11 $(\mathrm{scFv})$. In negative ion mode, the protein and the nonspecific complexes were detected predominantly in their deprotonated form, i.e., $(\mathrm{P}-\mathrm{nH})^{\mathrm{n}-} \equiv \mathrm{P}^{\mathrm{n}-}$ and $(\mathrm{P}+$ 1-nH $)^{\mathrm{n}-} \equiv(\mathrm{P}+\mathbf{1})^{\mathrm{n}-}$, at charge states $n=3-5(\mathrm{Ubq}), 6$ and $7(\mathrm{Lyz})$ or 8 and $9(\mathrm{scFv})$. The lower protein charge states observed in negative ion mode, compared to positive ion mode, likely reflect the greater effectiveness of acetic acid to compete with the deprotonated proteins for charge in negative ion mode relative to the ability of ammonia to compete with the protonated proteins for charge in positive ion mode [24].

Shown in Figure 2 are the normalized distributions of 1 bound nonspecifically to each pair of proteins investigated, as determined from the ES mass spectra. Importantly, in both positive and negative ion modes, the distributions are indistinguishable, within experimental error. The equivalency in the measured distributions is, perhaps, more clearly seen from a comparison of the corresponding $f_{i, P}$ values, the fractional abundance of the $(\mathrm{P}+i \mathrm{~L})$ species, where $\mathrm{P}=\mathrm{Ubq}$, Lyz, or scFv and $i$ is the number of molecules of $\mathrm{L}(=\mathbf{1 - 4})$ bound nonspecifically to the proteins, which were calculated using eq 3 :

$$
f_{i, P}=\frac{\sum_{n} A b\left(P L_{i}^{n+/-}\right)}{\sum_{i} \sum_{n} A b\left(P L_{i}^{n+/-}\right)}
$$

Listed in Table 1 are the ratios of the calculated $f_{i, P}$ values determined for each pair of proteins and $\mathbf{1}$ from replicate measurements performed in both positive and negative ion modes. Notably, within experimental error, the ratios are equal to 1.0 in all cases. These results suggest that, regardless of the polarity of the ES-MS measurements, nonspecific binding of acidic carbohy- 


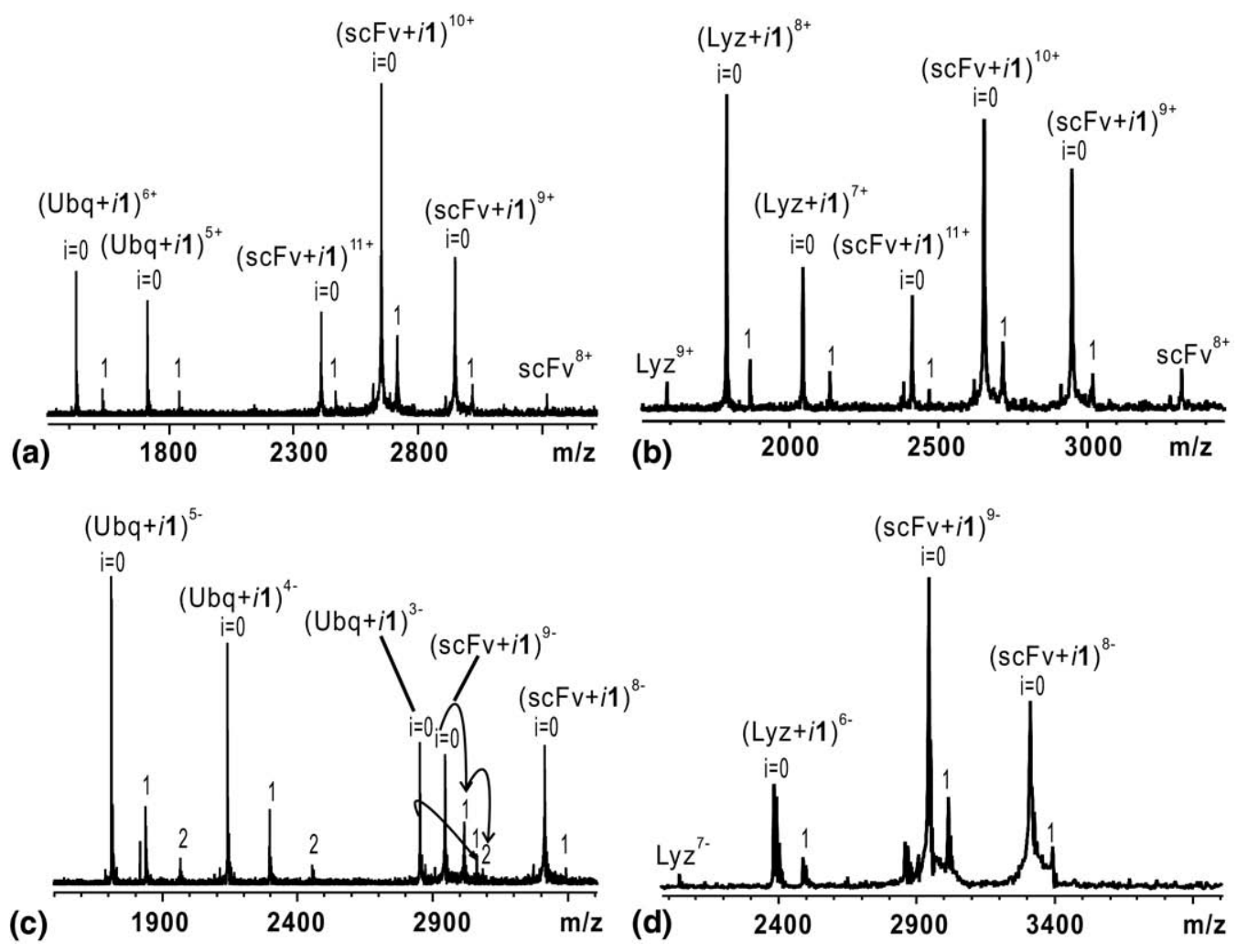

Figure 1. NanoES mass spectra obtained for solutions of $\mathbf{1}(106 \mu \mathrm{M})$ with (a) Ubq $(7 \mu \mathrm{M})$ and scFv $(10 \mu \mathrm{M})$, or (b) Lyz $(10 \mu \mathrm{M})$ and scFv $(10 \mu \mathrm{M})$ in positive ion mode, or with (c) Ubq $(6 \mu \mathrm{M})$ and scFv $(14 \mu \mathrm{M})$, or (d) Lyz $(12 \mu \mathrm{M})$ and scFv $(14 \mu \mathrm{M})$ in negative ion mode. The number of molecules of 1 bound to the protein ions is indicated by $i$.

drates, such as 1, to proteins in ES is independent of the size and structure of the proteins. These findings are consistent with those previously reported for neutral carbohydrates $[8,18]$.
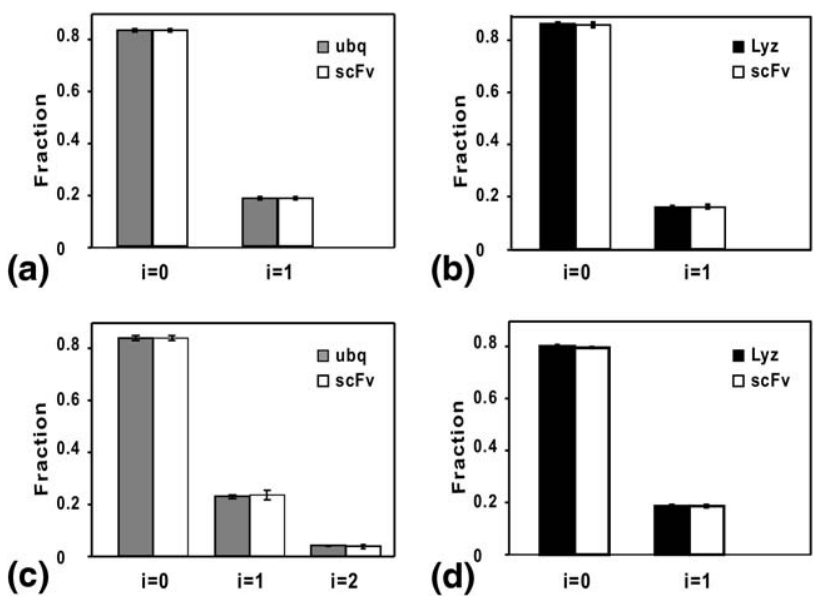

Figure 2. Distribution of nonspecific $(\mathrm{P}+i \mathbf{1})$ complexes for (a) $\mathrm{P}=\mathrm{Ubq}$ and $\mathrm{scFv}$, and $(\mathbf{b}) \mathrm{P}=\mathrm{Lyz}$ and $\mathrm{scFv}$, as determined from ES mass spectra measured in positive ion mode, or (c) $\mathrm{P}=\mathrm{Ubq}$ and $\mathrm{scFv}$, and (d) P = Lyz and scFv, as determined from ES mass spectra measured in negative ion mode. The reported errors correspond to one standard deviation and were determined from five replicate measurements.
To test whether the findings described above are general for acidic molecules, ES-MS measurements were performed on solutions containing pairs of the model proteins and 2, a weak acid with a $\mathrm{pK}_{\mathrm{a}}$ of 6.5 [25] (Figure S1, supplementary data, which can be found in the electronic version of this article). Qualitatively, the mass spectra obtained for solutions of $\mathbf{2}$ are similar to those obtained for $\mathbf{1}$. The measured distributions of 2 bound to each pair of proteins are shown in Figure S2 and the ratios of the corresponding $f_{i, p}$ values are listed in Table 1. It can be seen that in positive ion mode, equivalent distributions were obtained for both pairs of proteins investigated. However, clear differences in the distributions are evident in negative ion mode. In both cases, 2 was found to bind more extensively to Lyz than $\mathrm{Ubq}$ or scFv. For example, values of 0.76 and 1.40 were determined for the $f_{0, L y z} / f_{0, s c F v}$ and $f_{0, \text { Ubq }} / f_{0, L y z}$ ratios, respectively.

\section{Basic Molecules}

ES-MS was also performed on solutions containing the model proteins and either 3 , which has a $\mathrm{pK}_{\mathrm{b}}$ of 1.52 [26], or 4, which contains three strongly basic arginine residues. Illustrative ES mass spectra acquired in positive and negative ion mode for solutions of $\mathrm{Ubq}$ and Lyz or Lyz and scFv with 3 or $\mathbf{4}$ are shown in Figure 3 
Table 1. Comparison of the ratio of $f_{i, P}$ terms determined for the nonspecific binding of L (1-4) to pairs of proteins (Ubq/Lyz, $\mathrm{Ubq} / \mathrm{scFv}$, and $\mathrm{Lyz} / \mathrm{scFv}$ ) in positive and negative ion mode $\mathrm{ES}-\mathrm{MS}^{\mathrm{a}, \mathrm{b}}$

\begin{tabular}{|c|c|c|c|c|c|c|c|c|}
\hline [Ubq] $(\mu \mathrm{M})$ & {$[\mathrm{Lyz}](\mu \mathrm{M})$} & {$[\mathrm{scFv}](\mu \mathrm{M})$} & {$[\mathrm{L}](\mu \mathrm{M})$} & Polarity & $f_{O, P 1} / f_{O, P 2}$ & $f_{1, P 1} / f_{1, P 2}$ & $f_{O, P 1} / f_{O, P 2}$ & $f_{1, P 1} / f_{1, P 2}$ \\
\hline & & & 1 & & $f_{O, \mathrm{Ubq}} / f_{O, \mathrm{scFv}}$ & $f_{1, \mathrm{Ubq}} / f_{1, \mathrm{scFv}}$ & $f_{O, \mathrm{Lyz}} / f_{O, \mathrm{scFv}}$ & $f_{1, \mathrm{Lyz}} / f_{1, \mathrm{scFv}}$ \\
\hline \multirow[t]{2}{*}{7} & & 10 & 106 & + & $1.00 \pm 0.01$ & $1.00 \pm 0.04$ & & \\
\hline & 10 & 10 & 106 & + & & & $1.00 \pm 0.01$ & $0.98 \pm 0.04$ \\
\hline \multirow[t]{2}{*}{6} & & 14 & 106 & - & $1.00 \pm 0.02$ & $0.98 \pm 0.08$ & & \\
\hline & 12 & 14 & $\begin{array}{r}106 \\
2\end{array}$ & - & $f_{o, \mathrm{Ubq}} / f_{o, \mathrm{Lyz}}$ & $f_{1, \mathrm{Ubq}} / f_{1, \mathrm{Lyz}}$ & $\begin{array}{c}1.00 \pm 0.01 \\
f_{O, \mathrm{Lyz}} / f_{O, \mathrm{scFv}}\end{array}$ & $\begin{array}{c}0.99 \pm 0.04 \\
f_{1, \mathrm{Lyz}} / f_{1, \mathrm{scFv}}\end{array}$ \\
\hline \multirow[t]{2}{*}{6} & 10 & & 100 & + & $1.00 \pm 0.01$ & $1.0 \pm 0.1$ & & \\
\hline & 10 & 10 & 100 & + & & & $1.00 \pm 0.01$ & $1.1 \pm 0.1$ \\
\hline \multirow[t]{3}{*}{3} & 10 & & 100 & - & $1.40 \pm 0.06$ & $0.45 \pm 0.09$ & & \\
\hline & 10 & 10 & 100 & - & & & $0.76 \pm 0.01$ & $1.25 \pm 0.04$ \\
\hline & & & 3 & & $f_{o, \mathrm{Ubq}} / f_{o, \mathrm{~L}}$ & $f_{1, \mathrm{Ubq}} / f_{1, \mathrm{Lyz}}$ & $f_{O, \mathrm{Lyz}} / f_{O, \mathrm{scFv}}$ & $f_{1, \mathrm{Lyz}} / f_{1, \mathrm{scFv}}$ \\
\hline \multirow[t]{3}{*}{10} & 10 & & 200 & + & $0.93 \pm 0.05$ & $1.3 \pm 0.2$ & & \\
\hline & 10 & 10 & 200 & + & & & $1.43 \pm 0.05$ & $0.63 \pm 0.05$ \\
\hline & 7 & 9 & 152 & + & & & $1.01 \pm 0.01^{c}$ & $0.95 \pm 0.04^{c}$ \\
\hline \multirow[t]{3}{*}{2} & 12 & & 141 & - & $1.00 \pm 0.01$ & $0.95 \pm 0.02$ & & \\
\hline & 10 & 10 & 200 & - & & & $0.95 \pm 0.03$ & $0.99 \pm 0.08$ \\
\hline & & & 4 & & $f_{o, \mathrm{Ubq}} / f_{O, \mathrm{Lyz}}$ & $f_{1, \mathrm{Ubq}} / f_{1, \mathrm{Lyz}}$ & $f_{O, \mathrm{Lyz}} / f_{O, \mathrm{scFv}}$ & $f_{1, \mathrm{Lyz}} / f_{1, \mathrm{scFv}}$ \\
\hline \multirow[t]{3}{*}{10} & 10 & & 49 & + & $0.93 \pm 0.05$ & $1.1 \pm 0.1$ & & \\
\hline & 10 & 10 & 49 & + & & & $1.4 \pm 0.1$ & $0.41 \pm 0.05$ \\
\hline & 10 & 10 & 82 & + & & & $1.01 \pm 0.01^{\mathrm{c}}$ & $0.94 \pm 0.03^{c}$ \\
\hline \multirow[t]{2}{*}{3} & 10 & & 66 & - & $1.00 \pm 0.01$ & $1.00 \pm 0.07$ & & \\
\hline & 6 & 15 & 66 & - & & & $0.99 \pm 0.01$ & $1.1 \pm 0.1$ \\
\hline
\end{tabular}

${ }^{\mathrm{a}} \mathrm{All}$ measurements performed at $25^{\circ} \mathrm{C}, \mathrm{pH} 7$.

batios calculated from average $f_{i, P}$ values taken from five measurements.

'Measurements performed on solutions containing $10 \mathrm{mM}$ imidazole.

and Figure S3, respectively. Shown in Figure 4 and Figure $\mathrm{S} 4$ are the measured distributions of nonspecifically bound 3 and 4 , respectively; the corresponding $f_{i, P}$ ratios are listed in Table 1. In contrast to the behavior described above for $\mathbf{2}$, the distributions measured for $\mathbf{3}$ and 4 in negative ion mode are equivalent for each pair of proteins, but are nonequivalent in positive ion mode. For both 3 and $\mathbf{4}$, there was a clear preference for nonspecific binding to $\mathrm{scFv}$ compared to Lyz, and a slight preference for Ubq over Lyz.

Taken together, the results obtained for 1-4 reveal that, depending on the polarity of the ES-MS measurements, the nonspecific association of small charged biomolecules to proteins during the ES process may be sensitive to the nature of protein. Importantly, in all of the cases investigated, equivalent distributions were observed when the polarity of the ES-MS measurements was opposite to that of the acid or base in solution. Based on these findings it is concluded that the $P_{\text {ref }}$ method for correcting ES mass spectra for nonspecific ligand-protein binding can be successfully extended to charged ligands by performing the ES-MS measurements in positive ion mode for the analysis of acidic (negatively charged) ligands and negative ion mode for the analysis of basic (positively charged) ligands. However, when the polarity of the measurements matches that of the ligand charge in solution, nonequivalent distributions may be observed. In such instances, the $\mathrm{P}_{\text {ref }}$ method will fail to provide a quantitative measure of the extent of nonspecific ligand binding. Below, the origin of the nonequivalent distributions is explored and a general strategy for the implementation of the $P_{\text {ref }}$ method for charged ligands, which relies on the use of stabilizing additives, is described.

\section{Influence of In-Source Dissociation}

In principle, the nonequivalent distributions measured for the nonspecifically bound acidic and basic biomolecules could arise in two ways. The nonequivalent distributions could result from differences in the sampling of the biomolecules by the offspring (progeny) droplets that ultimately lead to the different gas-phase protein ions. Alternatively, the nonequivalent distributions could also result from the differential dissociation of the nonspecific interactions in the gas phase. In other words, the ES process leads to equivalent distributions, independent of the polarity of the measurements or the charge state of the biomolecule in solution, but the distributions are perturbed by the dissociation of the nonspecific interactions in the ion source (i.e., in-source dissociation), with the extent of dissociation dependent on the nature (e.g., size, structure, and charge state) of the protein ions. To test whether differential gas-phase dissociation was, at least in part, responsible for the observation of nonequivalent distributions of nonspecifically bound 2-4, BIRD "snapshot" experiments were performed. In contrast to the normal BIRD experiments [27], reactant ions are not isolated in the snapshot experiments. Instead, all of the gas-phase protein ions, including the nonspecific complexes, produced by ES and introduced into the ion cell of the FT-ICR MS are 
(a)
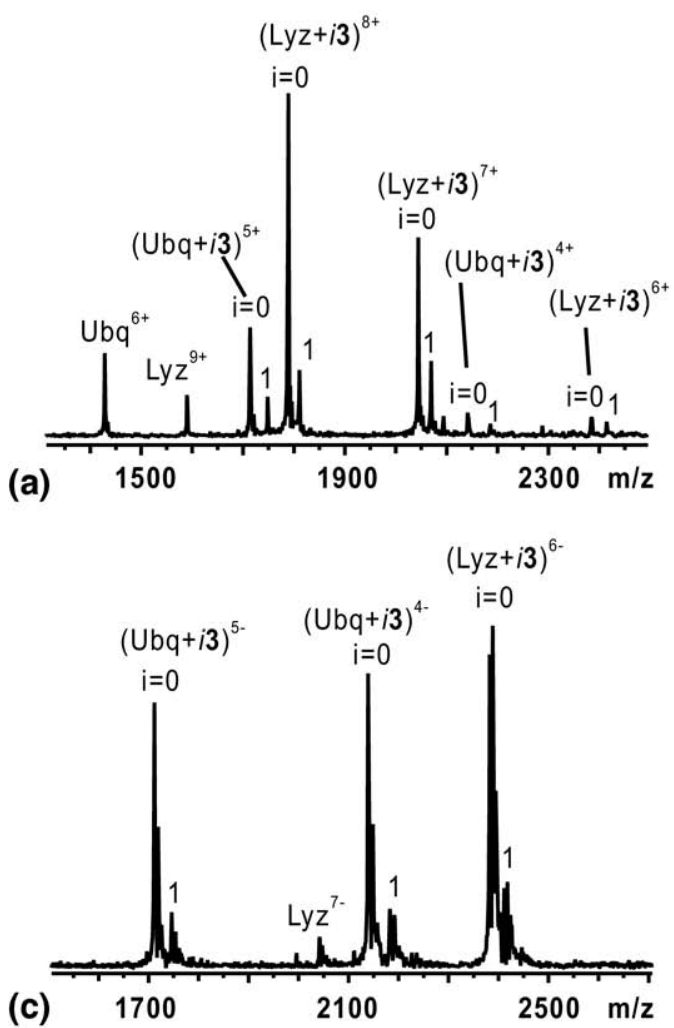
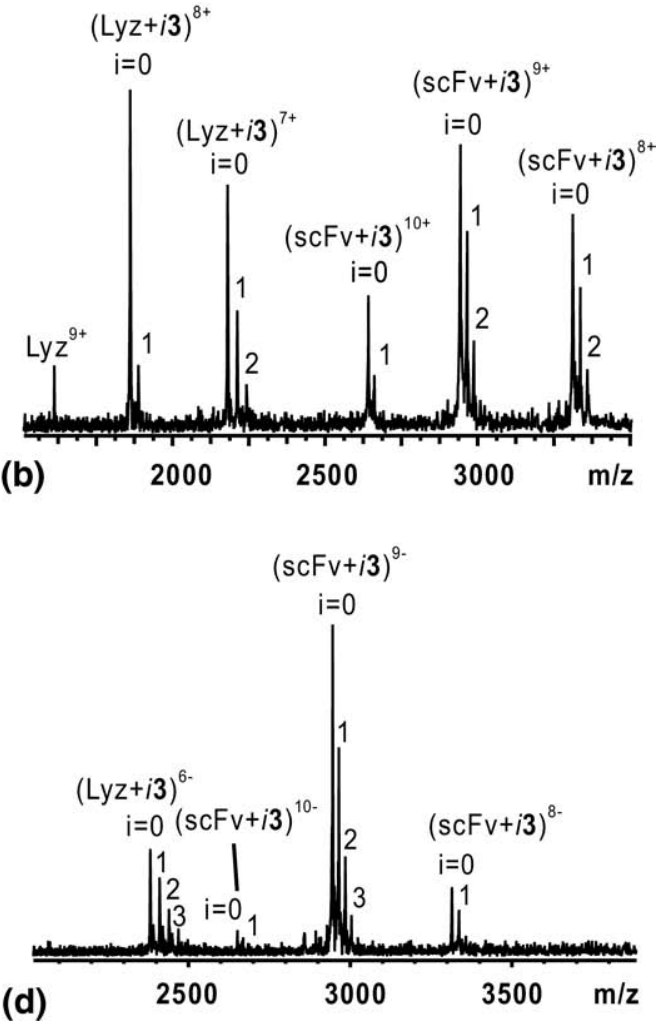

Figure 3. NanoES mass spectra obtained for solutions of (a) Ubq (10 $\mu \mathrm{M})$, Lyz $(10 \mu \mathrm{M})$, and 3 (200 $\mu \mathrm{M})$ and, (b) Lyz $(10 \mu \mathrm{M}), \mathrm{scFv}(10 \mu \mathrm{M})$, and $3(200 \mu \mathrm{M})$ in positive ion mode, and solutions of (c) Ubq $(2 \mu \mathrm{M}), \operatorname{scFv}(12 \mu \mathrm{M})$ and $3(141 \mu \mathrm{M})$ and, (d) Lyz $(10 \mu \mathrm{M}), \operatorname{scFv}(10 \mu \mathrm{M})$ and $3(200 \mu \mathrm{M})$ in negative ion mode. The number of molecules of $\mathbf{1}$ bound to the protein ions is indicated by $i$.

allowed to react [6]. In the present work, the BIRD snapshot experiments were performed at a cell temperature of $120^{\circ} \mathrm{C}$, which is similar to the estimated effective temperature of the proteins ions during accumulation in the hexapole of the ion source of the FT-ICR
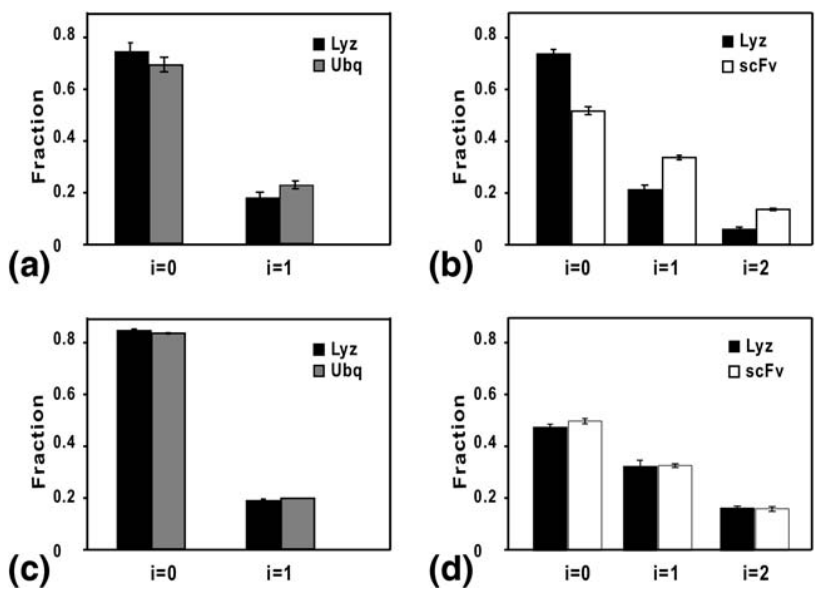

Figure 4. Distribution of nonspecific $(\mathrm{P}+i 3)$ complexes for (a) $\mathrm{P}=\mathrm{Ubq}$ and Lyz and (b) $\mathrm{P}=\mathrm{Lyz}$ and scFv, as determined from ES mass spectra measured in positive ion mode, or (c) $\mathrm{P}=\mathrm{Ubq}$ and Lyz and (d) Lyz and scFv as determined from ES mass spectra measured in negative ion mode. The reported errors correspond to one standard deviation and were determined from five replicate measurements.
MS used in this study [6], and a reaction time of $1 \mathrm{~s}$, which is comparable to the hexapole accumulation times typically used in the ES-MS measurements [6].

Shown in Figure 5a is an ES mass spectrum acquired in positive ion mode for a solution of $\mathrm{Lyz}, \mathrm{scFv}$, and 3; shown in Figure 5b is the corresponding BIRD snapshot mass spectrum. The distributions of nonspecifically bound 3 measured before and after BIRD are shown in Figure $5 \mathrm{c}$ and $\mathrm{d}$, respectively; the normalized distributions of charge states for Lyz and scFv are shown in Figure $5 \mathrm{e}$ and $\mathrm{f}$, respectively. Before BIRD, ions corresponding to free Lyz and $\mathrm{scFv}$ and to Lyz and $\mathrm{scFv}$ bound to one or two molecules of 3 were detected. After $1 \mathrm{~s}$ reaction at $120^{\circ} \mathrm{C}$, ions corresponding to the nonspecific complexes of $\mathrm{scFv}$ and 3 were still detected. However, the extent of nonspecific binding was reduced, compared with that measured before reaction, indicating that a fraction of the nonspecific complexes had dissociated. In the case of Lyz, only bare protein ions were detected after BIRD, indicating that all the nonspecific interactions had dissociated within $1 \mathrm{~s}$. Based on these results, it is concluded that the nonspecific interactions between 3 and Lyz formed in the ES process are less stable kinetically than those involving $\mathrm{scFv}$. As a result, the $(\mathrm{Lyz}+i 3)^{n+}$ ions are expected to be more prone to in-source dissociation than the corresponding $(\mathrm{scFv}+i 3)^{n+}$ ions. This difference in gas- 

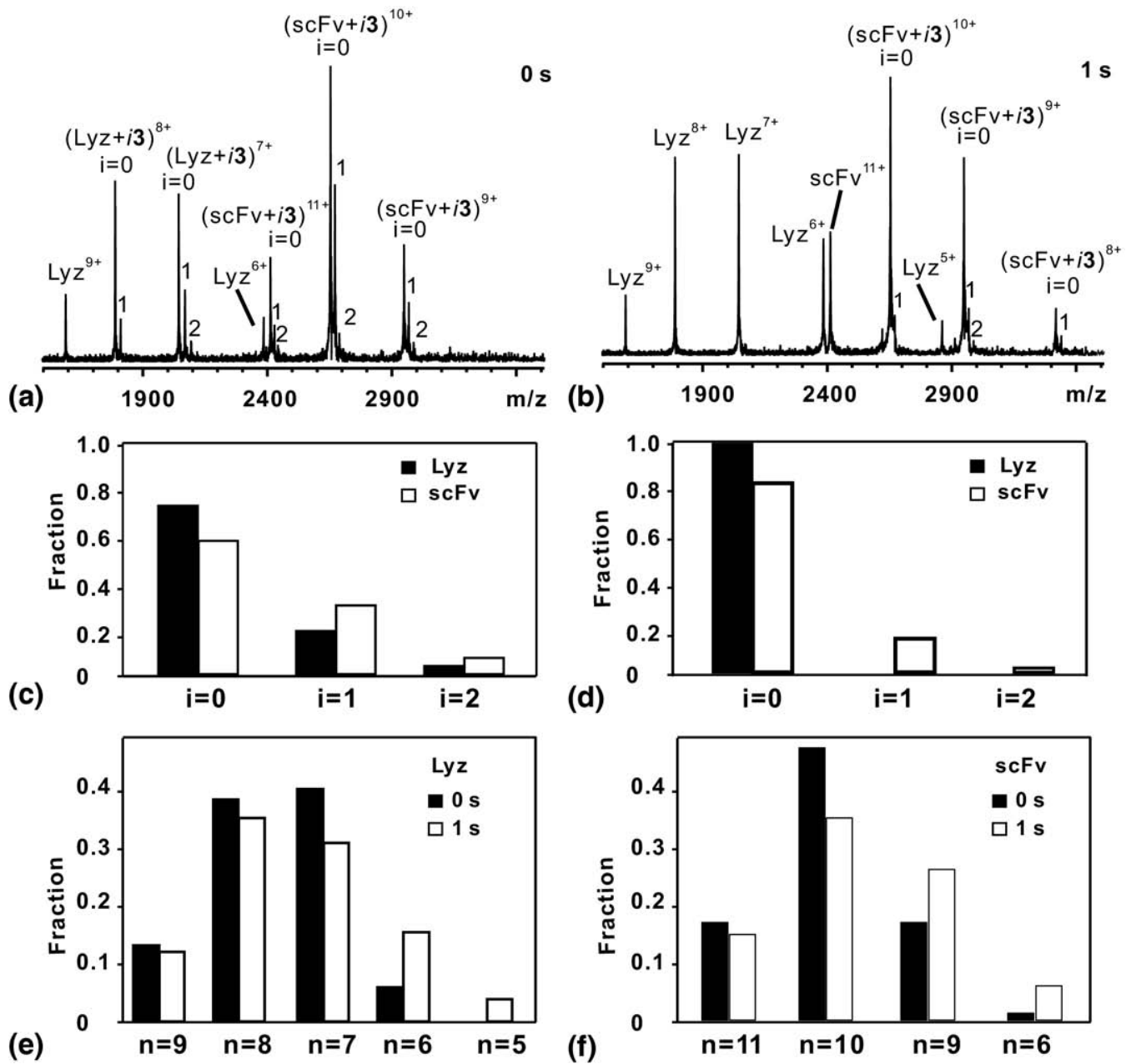

Figure 5. (a) NanoES mass spectrum obtained in positive ion mode for a solution of 3 (152 $\mu \mathrm{M})$, Lyz $(10 \mu \mathrm{M})$, and $\mathrm{scFV}(10 \mu \mathrm{M}),(\mathbf{b})$ BIRD snapshot mass spectrum obtained for solution (a) at cell temperature of $120^{\circ} \mathrm{C}$ and a reaction time of $1 \mathrm{~s}$. (c) and (d) Distribution of nonspecific $(\mathrm{P}+i 3)$ complexes for $\mathrm{P}=\mathrm{Lyz}$ and $\mathrm{scFv}$, as determined from ES mass spectra shown in (a) and (b), respectively. (e) Charge state distributions for $(\mathrm{Lyz}+i 3)^{n+}$ ions determined from ES mass spectra shown in (a) and (b). (f) Charge state distribution for $(\mathrm{scFv}+i 3)^{n+}$ ions determined from ES mass spectra shown in (a) and (b).

phase stability is consistent with the nonequivalent distributions measured for 3 bound to Lyz and to scFv. Similar comparative measurements were performed on the nonspecific protein complexes with 2 and with 4 . Shown Figure S5 and S6 are the corresponding mass spectra and normalized distributions measured in positive ion mode for a solution of $\mathrm{Lyz}, \mathrm{scFv}$ with 4 , and in negative ion mode for a solution of $\mathrm{Ubq}$, Lyz with 2, respectively. The results obtained for 4 are consistent with those described above for 3 , with the nonspecific interactions formed between 4 and $\mathrm{scFv}$ being more stable than those involving Lyz. However, the nonspecific interactions between 4 and $\mathrm{Lyz}$ or $\mathrm{scFv}$ are more stable (kinetically) than those involving 3, since interactions between 4 and Lyz are still evident after $1 \mathrm{~s}$ reaction. The results obtained for 2 revealed that the nonspecific interactions with Lyz are somewhat more stable than those with Ubq, although in both cases very little change in the extent of nonspecific binding was measured after $1 \mathrm{~s}$ reaction. Nevertheless, the greater kinetic stability of the interactions with Lyz is consistent with the observation of greater nonspecific binding of $\mathbf{2}$ to Lyz, compared to Ubq.

A second important finding was that BIRD resulted in a small but measurable decrease in the average charge states of the protein ions. The weighted average charge states $(A C S)$ of the different proteins (free and nonspecifically bound) were calculated from the ES mass spectra using eq 4 :

$$
A C S=\frac{\sum_{n} n \sum_{i} A b\left(P L_{i}^{n+/-}\right)}{\sum_{n} \sum_{i} A b\left(P L_{i}^{n+/-}\right)}
$$

For example, in the case of the nonspecific complexes of 3 with Lyz and with scFv, the ACS decreased from 7.5 to 7.2 for Lyz; for scFv the ACS decreased from 10.0 to 9.6. The decrease in the ACS indicates that a fraction of 
3 dissociated from the proteins in its protonated form. These results suggest that, in positive ion mode, a fraction of 3 is protonated and interacts with the proteins through ionic hydrogen bonds (e.g., (base + $\mathrm{H})^{+}$-protein). In such cases the strength of the nonspecific interactions will reflect, in part, the gas-phase basicity of the proteins ions, which is sensitive to protein structure and to Coulombic effects resulting from the multiple charges [28, 29]. Ionic hydrogen bonds may also stabilize the deprotonated complexes of 1 and 2 (e.g., (acid-H) ${ }^{-}$-protein). In such instances, the kinetic stabilities of the nonspecific interactions will be sensitive to the gas-phase acidity of the protein ions, which is similarly influenced by Coulombic effects. When the charge states of the protein and the acidic or basic biomolecule are of the same polarity in the gasphase, the nonspecific interactions will be destabilized by Coulombic repulsion and in-source dissociation, which can lead to nonequivalent distributions of nonspecifically bound charged molecules, will be promoted. The stabilities of the nonspecific complexes involving 1 are also expected to be sensitive to Coulombic effects. However, these nonspecific interactions are anticipated to be quite stable in the gas-phase given the large number of hydroxyl groups in $\mathbf{1}$ that can participate in intermolecular H-bonds. The observation of equivalent distributions observed for $\mathbf{1}$ in both positive and negative ion mode is attributed to the relatively high gas-phase stability of the nonspecific proteincarbohydrate interactions.

\section{Minimizing In-Source Dissociation of the Nonspecific Interactions}

The aforementioned results provide indirect evidence that the nonequivalent distributions of nonspecifically bound 2-4 observed when the polarity of the ES-MS measurement matched that of the acid or base in solution arise, at least in part, from differential insource dissociation of the nonspecific interactions. To test this hypothesis directly, the ES-MS measurements were performed in positive ion mode on solutions containing pairs of the model proteins and either 3 or 4 , in the presence of a stabilizing solution additive. Shown in Figure 6a is an illustrative ES mass spectrum measured for a solution of $3(152 \mu \mathrm{M})$, Lyz $(10 \mu \mathrm{M}), \mathrm{scFv}(10$ $\mu \mathrm{M})$, and imidazole $(10 \mathrm{mM})$. The presence of imidazole in solution has recently been shown to protect labile protein-ligand complexes from in-source dissociation in ES-MS [11, 30]. While the nature of the stabilizing effect of imidazole has not been conclusively estab- (a)
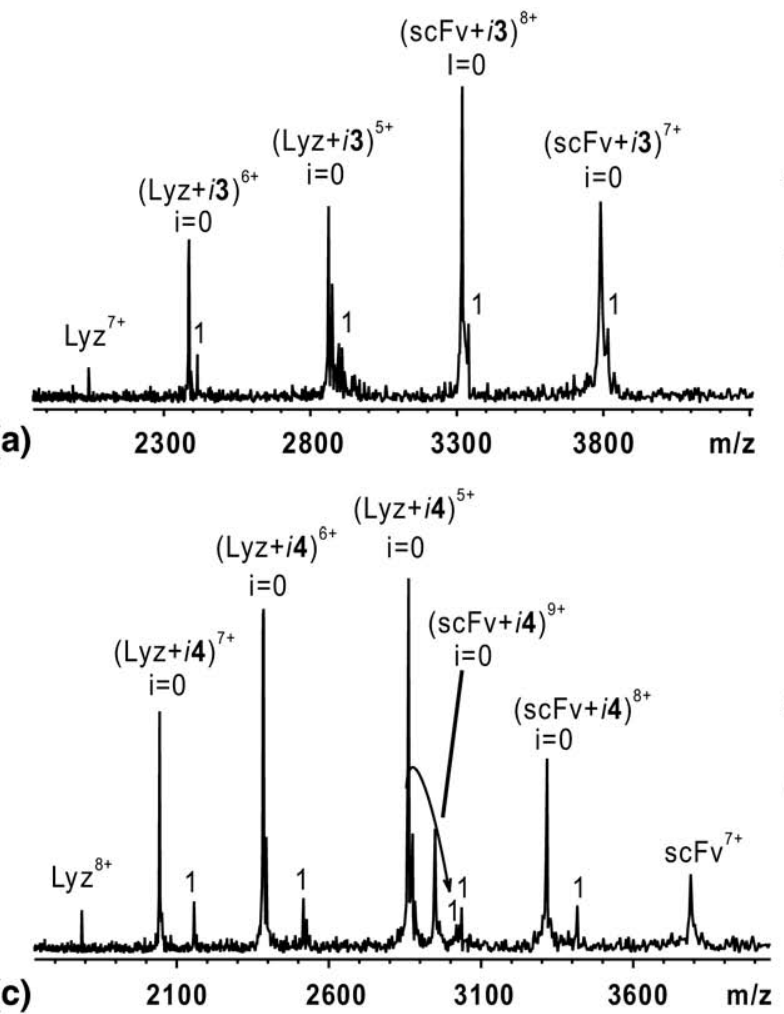
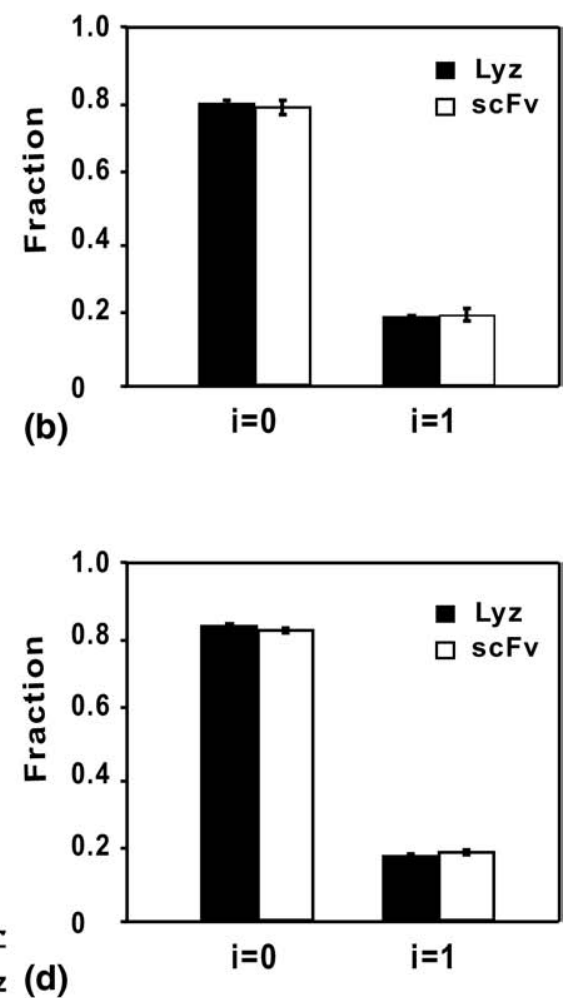

Figure 6. (a) NanoES mass spectrum obtained in positive ion mode for a solution of Lyz (10 $\mu \mathrm{M})$, scFv $(10 \mu \mathrm{M}), 3(152 \mu \mathrm{M})$, and imidazole $(10 \mathrm{mM})$. (b) Distribution of nonspecific $(\mathrm{P}+i 3)$ complexes, where $\mathrm{P}=\mathrm{Lyz}$ and $\mathrm{scFv}$, as determined from (a). (c) NanoES mass spectrum obtained in positive ion mode for a solution of (c) Lyz $(10 \mu \mathrm{M})$, scFv $(10 \mu \mathrm{M}), 4(82 \mu \mathrm{M})$, and imidazole $(10 \mathrm{mM})$. (d) Distribution of nonspecific $(\mathrm{P}+i 4)$ complexes, where $\mathrm{P}=\mathrm{Lyz}$ and $\mathrm{scFv}$, as determined from (c). The errors correspond to one standard deviation. 
lished, it is thought to arise, at least in part, from enhanced evaporative cooling resulting from the dissociation of multiple imidazole adducts of the proteinligand complexes in the ion source [11]. The introduction of imidazole to the solution is typically accompanied by a decrease in the absolute charge states of protein ions in both positive and negative ion mode ES-MS. For example, the ACS decreased from 7.57 to 5.40 for Lyz and from 9.48 to 7.58 for scFv. This charge shift effect, which can be explained by the relatively high gas-phase basicity $(217 \mathrm{kcal} / \mathrm{mol})$ [31] of imidazole, may also lead to more stable (kinetically) protein ions. Regardless of the origin of the stabilizing effect, the measured distributions of 3 bound to Lyz and scFv in the presence of imidazole are indistinguishable, Figure $6 \mathrm{~b}$. Importantly, equivalent distributions were also measured for the nonspecific association of 4 to Lyz and $\mathrm{scFv}$ (Figure $6 \mathrm{c}$ and $\mathrm{d}$ ) in the presence of imidazole. Listed in Table 1 are the ratios of the calculated $f_{i, P}$ values determined for each pair of proteins and $\mathbf{3}$ or $\mathbf{4}$ from replicate measurements performed in positive ion mode. Notably, the ratios are close to 1.0 in all cases.

These results confirm that the nonspecific association of small, charged biomolecules to proteins during ES is a random process, independent of the nature of the protein. These findings are consistent with the results previously reported for the nonspecific binding of neutral carbohydrates to proteins in ES-MS [8, 18]. Also, and as found with neutral carbohydrates [32], the kinetic stabilities of the nonspecific interactions involving acidic and basic biomolecules are sensitive to the nature of the protein ions and, in a given ES-MS experiment, in-source dissociation can lead to differences in the distributions of nonspecifically bound molecules measured for different proteins. However, the original distributions can be preserved by introducing imidazole, a stabilizing additive, to the ES solution.

\section{Conclusions}

The present study represents the first detailed investigation into the formation of nonspecific interactions between small acidic and basic biomolecules and proteins in the ES process. Control experiments revealed that the distributions of non-interacting (in solution) acidic and basic biomolecules bound nonspecifically to a given pair of proteins were generally sensitive to the polarity of the ES-MS measurements. In all cases, equivalent distributions were measured when the acidic and basic molecules were analyzed in positive and negative ion mode, respectively. In contrast, nonequivalent distributions were typically observed when the measurements were performed in the opposite polarity. The lone exception was the acidic trisaccharide $6^{\prime}$-sialyllactose, for which equivalent distributions were observed in both modes. This observation likely reflects the relatively high gasphase stability of the nonspecific interactions owing to the ability of the trisaccharide to form many intermolecular H-bonds. The results of time-resolved BIRD experiments showed that the kinetic stabilities of the nonspecific interactions are sensitive to the nature of the protein ions and suggested that differential gas-phase dissociation of the nonspecific interactions in the ion source was responsible for the observation of the nonequivalent distributions. This hypothesis was confirmed by the observation of equivalent distributions upon introduction of imidazole, a stabilizing additive known to protect protein complexes from in-source dissociation, to the solution. Taken together, the results of this study indicate that the nonspecific association of small, acidic and basic biomolecules to proteins during ES is a random process, independent of the nature of the protein. Consequently, it is concluded that the $P_{\text {ref }}$ method can be used to quantitatively monitor the occurrence of nonspecific binding of ionic ligands to proteins in ES-MS, provided that in-source dissociation of the nonspecific interactions is avoided. This condition is most easily satisfied by using a stabilizing solution additive. Alternatively, in-source dissociation may be suppressed by analyzing basic ligands using negative ion mode and acidic ligands using positive ion mode.

\section{Acknowledgments}

The authors acknowledge the Natural Sciences and Engineering Research Council of Canada and the Alberta Ingenuity Centre for Carbohydrate Science for funding.

\section{Appendix A Supplementary Material}

Supplementary material associated with this article may be found in the online version at doi:10.1016/ j.jasms.2009.12.002.

\section{References}

1. Loo, J. A. Studying Noncovalent Protein Complexes by Electrospray Ionization Mass Spectrometry. Mass Spectrom. Rev. 1997, 16, 1-23.

2. Yu, Y. H.; Kirkup, C. E.; Pi, N.; Leary, J. A. Characterization of Noncovalent Protein-Ligand Complexes and Associated Enzyme Intermediates of GlcNAc-6-O-Sulfotransferase by Electrospray Ionization FT-ICR Mass Spectrometry. J. Am. Soc. Mass Spectrom. 2004, 15, 14001407.

3. Heck van den Heuvel, R. H. H.; Heck, A. J. R. Investigation of Intact Protein Complexes by Mass Spectrometry. Mass Spectrom. Rev. 2004, 23, 368-389.

4. Jorgensen, T. J. D.; Roepstorff, P.; Heck, A. J. R. Direct Determination of Solution Binding Constants for Noncovalent Complexes between Bacterial Cell Wall Peptide Analogues and Vancomycin Group Antibiotics by Electrospray Ionization Mass Spectrometry. Anal. Chem. 1998, 70, 4427-4432.

5. Daniel, J. M.; Friess, S. D.; Rajagopalan, S.; Wendt, S.; Zenobi, R. Quantitative Determination of Noncovalent Binding Interactions Using Soft Ionization Mass Spectrometry. Int. J. Mass Spectrom. 2002, 216, 1-27.

6. Wang, W.; Kitova, E. N.; Klassen, J. S. Influence of Solution and Gas Phase Processes on Protein-Carbohydrate Binding Affinities Determined by Nanoelectrospray Fourier Transform Ion Cyclotron Resonance Mass Spectrometry. Anal. Chem. 2003, 75, 4945-4955.

7. Gabelica, V.; Galic, N.; Rosu, F.; Houssier, C.; De Pauw, E. Influence of Response Factors on Determining Equilibrium Association Constants of Noncovalent Complexes by Electrospray Ionization Mass Spectrometry. J. Mass Spectrom. 2003, 38, 491-501.

8. Wang, W.; Kitova, E. N.; Klassen, J. S. Nonspecific ProteinCarbohydrate Complexes Produced by Nanoelectrospray Ionization. Factors Influencing Their Formation and Stability. Anal. Chem. 2005, 77, 3060-3071. 
9. Robinson, C. V.; Chung, E. W.; Kragelund, B. B.; Knudsen, J.; Aplin, R. T.; Poulsen, F. M.; Dobson, C. M. Probing the Nature of Noncovalent Interactions by Mass Spectrometry. A Study of Protein-CoA Ligand Binding and Assembly. J. Am. Chem. Soc. 1996, 118, 8646-8653.

10. Clark, S. M.; Konermann, L. Determination of Ligand-Protein Dissociation Constants by Electrospray Mass Spectrometry-Based Diffusion Measurements. Anal. Chem. 2004, 76, 7077-7083.

11. Sun, J.; Kitova, E. N.; Klassen, J. S. Method for Stabilizing ProteinLigand Complexes in Nanoelectrospray Ionization Mass Spectrometry. Anal. Chem. 2007, 79, 416-425.

12. Liu, L.; Bagal, D.; Kitova, E. N.; Klassen, J. S. Hydrophobic ProteinLigand Interactions Preserved in the Gas Phase. I. Am. Chem. Soc. 2009, $131,15980-15981$

13. Xie, Y.; Zhang, J.; Yin, S.; Loo, J. A. Top-Down ESI-ECD-FT-ICR Mass Spectrometry Localizes Noncovalent Protein-Ligand Binding Sites. J. Am. Chem. Soc. 2006, 128, 14432-14433.

14. Kitova, E. N.; Seo, M.; Roy, P. N.; Klassen, J. S. Elucidating the Intermolecular Interactions within A Desolvated Protein-ligand Complex. An Experimental and Computational Study. J. Am. Chem. Soc. 2008, 130, 1214-1226

15. Bagal, D.; Kitova, E. N.; Liu, L.; El-Haweit, A.; Schnier, P. D.; Klassen, J. S. Gas-Phase Stabilization of Noncovalent Protein Complexes Formed by Electrospray Ionization. Anal. Chem. 2009, 81, 7801-7806.

16. Sun, J.; Kitova, E. N.; Sun, N.; Klassen, J. S. Method for Identifying Nonspecific Protein-Protein Interactions in Nanoelectrospray Ionization Mass Spectrometry. Anal. Chem. 2007, 79, 8301-8311.

17. Sun, N.; Sun, J.; Kitova, E. N.; Klassen, J. S. Identifying Nonspecific Ligand Binding in Electrospray Ionization Mass Spectrometry Using the Reporter Molecule Method. J. Am. Soc. Mass Spectrom. 2009, 20, 1242 1250.

18. Sun J.; Kitova, E. N.; Wang, W.; Klassen, J. S. Method for Distinguishing Specific from Nonspecific Protein-Ligand Complexes in Nanoelectrospray Ionization Mass Spectrometry. Anal. Chem. 2006, 78, 3010-3018.

19. Shoemaker, G. K.; Soya, N.; Palcic, M. M.; Klassen, J. S. TemperatureDependent Cooperativity In Donor-Acceptor Substrate Binding to Human Blood Group Glycosyltransferases. Glycobiology 2008, 18, 587592.

20. Kitova, E. N.; Kitov, P. I.; Paszkiewicz, E.; Kim, J.; Mulvey, G. L.; Armstrong, G. D.; Bundle, D. R.; Klassen, J. S. Affinities of Shiga Toxins 1 and 2 for Univalent and Oligovalent Pk Trisaccharide Analogs Measured by Electrospray Ionization Mass Spectrometry. Glycobiology 2007, 17, 1127-1137.
21. Touboul, D.; Maillard, L.; Grasslin, A.; Moumne, R.; Seitz, M.; Robinson, J.: Zenobi, R. How to Deal with Weak Interactions in Noncovalent Complexes Analyzed by Electrospray Mass Spectrometry: Cyclopeptidic Inhibitors of the Nuclear Receptor Coactivator 1-STAT6. J. Am. Soc. Mass Spectrom. 2009, 20, 303-311.

22. Zdanov, A.; Bundle, D. R.; Deng, S.-J.; MacKenzie, C. R.; Narang, S. A. Young, M. N.; Cygler, M. Structure of A Single-Chain Antibody Variable Domain (Fv) Fragment Complexed with A Carbohydrate Antigen at 1.7- $\AA$ Resolution. Proc. Natl. Acad. Sci. U.S.A. 1994, 91 6423-6427.

23. Leavell, M. D.; Leary, J. A. Stabilization and Linkage Analysis of Metal-Ligated Sialic Acid Containing Oligosaccharides. J. Am. Soc. Mass Spectrom. 2001, 12, 528-536.

24. Verkerk, U. H.; Peschke, M.; Kebarle, P. Effect of Buffer Cations and of $\mathrm{H}_{3} \mathrm{O}^{+}$on the Charge States of Native Proteins. Significance to Determinations of Stability Constants of Protein Complexes. J. Mass Spectrom. 2003, 38, 618-631.

25. Dawson, R. M. C.; Elliott, W. H.; Jones, K. M. Data for Biochemical Research; Clarendon Press: Oxford, 1986; p. 113.

26. Voet, D.; Voet, J. G.; Pratt, C. W. Fundamentals of Biochemistry; John Wiley and Sons, 2002; p. 81.

27. Price, W. D.; Schnier, P. D.; Williams, E. R. Tandem Mass Spectrometry of Large Biomolecule Ions by Blackbody Infrared Radiative Dissociation. Anal. Chem. 1996, 68, 859-866.

28. Meot-Ner, M. The Ionic Hydrogen Bond and Ion Solvation. 1. $\mathrm{NH}^{+} \cdots \mathrm{O}$ $\mathrm{NH}^{+} \ldots \mathrm{N}$, and $\mathrm{OH}^{+} \ldots \mathrm{O}$ Bonds. Correlations with Proton Affinity Deviations due to Structural Effects. J. Am. Chem. Soc. 1984, 106, 1257-1264.

29. Gross, D. S.; Williams, E. R. Experimental Measurement of Coulomb Energy and Intrinsic Dielectric Polarizability of a Multiply Protonated Peptide Ion Using Electrospray Ionization Fourier-Transform Mass Spectrometry. J. Am. Chem. Soc. 1995, 117, 883-890.

30. van Dujin, E.; Barendregt, A.; Synowsky, S.; Versluis, C.; Heck, A. J. R. Chaperonin Complexes Monitored by Ion Mobility Spectrometry. J. Am. Chem. Soc. 2009, 131, 1452-1459.

31. Hunter, E. P.; Lias, S. G. Evaluated Gas Phase Basicities and Proton Affinities of Molecules: An Update. Phys. Chem. Ref. Data 1998, 27, (3), 413-656.

32. Wang, W.; Kitova, E. N.; Klassen, J. S. Blackbody Infrared Radiative Dissociation of Nonspecific Protein-Carbohydrate Complexes Produced by Nanoelectrospray Ionization: The Nature of the Noncovalent Interactions. J. Am. Soc. Mass Spectrom. 2005, 16, 1583-1594. 